\title{
INTERSECTION NUMBERS FOR LOADED CYCLES ASSOCIATED WITH SELBERG-TYPE INTEGRALS
}

\author{
Dedicated to Professor Atsushi Inoue on his sixtieth birthday
}

\author{
Katsuhisa Mimachi, Katsuyoshi OHaRa and MasaAKi Yoshida
}

(Received March 27, 2003)

Abstract. We evaluate the intersection numbers of loaded cycles associated with an $n$-fold Selberg-type integral. We proceed inductively using high-dimensional local systems.

Introduction. We evaluate the intersection numbers of loaded cycles associated with the $n$-fold Selberg-type integral

$$
\int u(t) d t_{1} \wedge \cdots \wedge d t_{n}, \quad u=\prod_{i=1}^{n} \prod_{(i<) j}\left(t_{i}-t_{j}\right)^{\alpha_{i j}}
$$

where $t_{j}(n<j)$ are mutually distinct parameters. Let $\mathcal{L}=\mathcal{L}^{+}$be the local system defined by the integrand $u$ on

$$
X^{n}=\left\{t=\left(t_{1}, \ldots, t_{n}\right) \in C^{n} \mid t_{i} \neq t_{j}, \quad 1 \leq i \leq n, 1 \leq j \neq i\right\},
$$

and $\mathcal{L}^{-}$its dual, i.e., the local system on $X$ defined by $u^{-1}$. When $t_{j}(n<j)$ are real, the real locus $X_{\boldsymbol{R}}^{n}$ of $X^{n}$ breaks into disjoint $n$-cells. We load each cell, say $\Delta$, with $u^{ \pm 1}$ in the standard way (see $\S 1.4$ ) and make it loaded cycles $\Delta_{ \pm}$, that is, elements of the locally finite $n$-th homology group $H_{n}^{\text {lf }}\left(X, \mathcal{L}^{ \pm}\right)$with coefficients in $\mathcal{L}^{ \pm}$. There is a natural dual pairing

$$
H_{n}^{\mathrm{lf}}(X, \mathcal{L}) \times H_{n}\left(X, \mathcal{L}^{-}\right) \rightarrow C
$$

called the intersection pairing. Throughout this paper we assume that the exponents $\alpha_{i j}$ are sufficiently generic so that the natural map

$$
H_{n}(X, \mathcal{L}) \rightarrow H_{n}^{\mathrm{lf}}(X, \mathcal{L})
$$

is an isomorphism; the inverse map is called the regularization and is denoted by reg. For general background, refer to [Yo].

The main purpose of this paper is the evaluation of the intersection numbers of these cycles. The so-called half-turn formula for these cycles plays a crucial role; this formula is important for its own sake. To help the reader's understanding, we present the context in $n=1,2,3$ before stating in general $n$.

2000 Mathematics Subject Classification. Primary 34M35; Secondary 33C70.

Key words and phrases. Twisted (co)homology, loaded cycles, intersection numbers, hypergeometric integrals, Selberg-type integrals, fibre bundle. 
We proceed inductively by utilizing a fibre bundle structure of the space $X^{n}$. In order to fit the notation of the coordinates and the points to induction, we fix them as follows. For each positive integer $n$ and a sequence of mutually distinct numbers

$$
t_{0}, t_{n+1}, t_{n+2}, \ldots,
$$

we consider a domain in $\boldsymbol{C}^{n}$ defined by

$$
X_{t_{0}, t_{n+1}, \ldots}^{1 \cdots n}:=\left\{\left(t_{1}, \ldots, t_{n}\right) \in C^{n} \mid t_{i} \neq t_{j}, 1 \leq i \leq n, 0 \leq j \neq i\right\} .
$$

The local system $\mathcal{L}$ (of rank 1 ) on $X_{t_{0}, t_{n+1}, \ldots}^{1 \cdots n}$ is defined by the function

$$
u:=\prod_{i=1}^{n}\left(t_{i}-t_{0}\right)^{\alpha_{i 0}} \prod_{(i<) j}\left(t_{i}-t_{j}\right)^{a_{i j}}
$$

on $X_{t_{0}, t_{n+1}, \cdots}^{1 \cdots n}$. Via the projection

$$
\pi: X_{t_{0}, t_{n+1}, \ldots}^{1 \cdots n} \ni\left(t_{1}, \ldots, t_{n}\right) \longmapsto t_{n} \in X_{t_{0}, t_{n+1}, \ldots}^{n}:=\left\{t_{n} \mid t_{n} \neq t_{0}, t_{n+1}, \ldots\right\},
$$

the space $X_{t_{0}, t_{n+1}}^{1 \cdots n}$ can be regarded as a fibre bundle over the 1 -dimensional space $X_{t_{0}, t_{n+1}, \ldots}^{n}$ with fibre

$$
\pi^{-1}\left(t_{n}\right)=X_{t_{0}, t_{n}, \ldots}^{1 \cdots(n-1)}=\left\{\left(t_{1}, \ldots, t_{n-1}\right) \mid t_{i} \neq t_{j}, 1 \leq i \leq n-1,0 \leq j\right\} .
$$

Making use of this fibre bundle structure, we express the intersection numbers of $n$-dimensional cycles on $X_{t_{0}, t_{n+1}, \ldots}^{1 \cdots n}$ in terms of those of $k$-dimensional cycles on $X_{t_{0}, t_{k+1}, \ldots}^{1 \cdots k}$ for $1 \leq k \leq$ $n-1$.

We name the cycles on $X_{t_{0}, t_{n}, \ldots}^{1 \cdots(n-1)}$ in $\S 1$, and the half-turn formula, which is the key of our method, is given in $\S 2$. Finally in $\S 3$, we give formulae for the intersection numbers. An application of these formulae is given in $\$ 4$. $\$ 5$ recalls a fundamental structure of twisted (co)homology groups of fibre bundles stated in [OST].

1. Coding the cycles. For indeterminates $t_{0}, t_{1}, \ldots$, we consider domains in real $t$ spaces.

1.1 1D case. On the real $t_{1}$-space, we fix $t_{0}<t_{2}<t_{3}<\cdots$ and name the intervals (see Figure 1)

$$
D_{0 \tilde{1} 2 \ldots}=D_{0 \tilde{1} 2}:=\left\{t_{1} \in \boldsymbol{R} \mid t_{0}<t_{1}<t_{2}\right\}, \quad D_{02 \tilde{1} 3 \ldots}=D_{2 \tilde{1} 3}:=\left\{t_{1} \mid t_{2}<t_{1}<t_{3}\right\}, \ldots
$$

( $\tilde{1}$ indicates that $t_{1}$ is a variable, i.e., not fixed.) We array these intervals as

$$
\begin{aligned}
& D_{023 \ldots}^{1}:={ }^{t}\left(D_{0 \tilde{1} 2}, D_{2 \tilde{1} 3}, D_{3 \tilde{1} 4}, \ldots\right) . \\
& \underset{t_{0}}{\stackrel{D_{0 \tilde{1} 2}}{t_{2}}{ }^{D_{2 \tilde{1} 3}}} \underbrace{}_{t_{3}} \cdot t_{1}
\end{aligned}
$$

FIGURE 1. Coding the intervals. 
1.2 2D case. On the real $\left(t_{1}, t_{2}\right)$-space, we fix $t_{0}<t_{3}<t_{4}<\cdots$ and name the domains (see Figure 2)

$D_{0 \tilde{1} \tilde{2} 3}:=\left\{\left(t_{1}, t_{2}\right) \in \boldsymbol{R}^{2} \mid t_{0}<t_{1}<t_{2}<t_{3}\right\}, D_{0 \tilde{2} \tilde{1} 3}:=\left\{\left(t_{1}, t_{2}\right) \mid t_{0}<t_{2}<t_{1}<t_{3}\right\}$,

$D_{0 \tilde{2} 3 \tilde{1}_{4}}:=\left\{\left(t_{1}, t_{2}\right) \mid t_{0}<t_{2}<t_{3}<t_{1}<t_{4}\right\}, D_{0 \tilde{1} 3 \tilde{2}_{4}}:=\left\{\left(t_{1}, t_{2}\right) \mid t_{0}<t_{1}<t_{3}<t_{2}<t_{4}\right\}, \ldots$.

( $\tilde{1}$ and $\tilde{2}$ indicate that $t_{1}$ and $t_{2}$ are variables, i.e., not fixed.) We array these domains as

$$
D_{034 \ldots}^{12}:={ }^{t}\left(D_{0 \tilde{2} 34 \ldots}^{1}, D_{03 \tilde{2} 4 \ldots}^{1}, D_{034 \tilde{2} 5 \ldots}^{1} \ldots\right),
$$

where

$$
D_{0 \tilde{2} 34 \ldots}^{1}:={ }^{t}\left(D_{0 \tilde{1} \tilde{2} 3}, D_{0 \tilde{2} \tilde{1} 3}, D_{0 \tilde{2} 3 \tilde{1} 4}, D_{0 \tilde{2} 341 \tilde{1}}, \ldots\right), \ldots
$$

1.3 3D case. On the real $\left(t_{1}, t_{2}, t_{3}\right)$-space, we fix $t_{0}<t_{4}<t_{5}<\cdots$ and name the domains

$$
D_{0 \tilde{1} \tilde{2} \tilde{3} 4 \ldots}:=\left\{\left(t_{1}, t_{2}, t_{3}\right) \in \boldsymbol{R}^{2} \mid t_{0}<t_{1}<t_{2}<t_{3}<t_{4}\right\}, \ldots .
$$

$\left(\tilde{1}, \tilde{2}, \tilde{3}\right.$ indicate that $t_{1}, t_{2}, t_{3}$ are variables, i.e., not fixed.) We array these intervals as

$$
D_{045 \ldots}^{123}:={ }^{t}\left(D_{0 \tilde{3} 4 \ldots}^{12}, D_{04 \tilde{3} 5 \ldots}^{12}, D_{045 \tilde{3} 6 \ldots}^{12}, \ldots\right), \ldots,
$$

where

$$
D_{0 \tilde{3} 4 \ldots}^{12}:={ }^{t}\left(D_{0 \tilde{2} \tilde{3} 4 \ldots}^{1}, D_{0 \tilde{2} \tilde{2} 4 \ldots}^{1}, D_{0 \tilde{3} 4 \tilde{2} 5 \ldots}^{1}, \cdots\right), \ldots
$$

and

$$
D_{0 \tilde{2} \tilde{3} 4 \ldots}^{1}:={ }^{t}\left(D_{0 \tilde{1} \tilde{2} \tilde{3} 4}, D_{0 \tilde{2} \tilde{1} \tilde{3} 4}, D_{0 \tilde{2} \tilde{z} \tilde{1} 4}, D_{0 \tilde{2} \tilde{3} 4 \tilde{1} 5}, \cdots\right), \ldots
$$

$1.4 n \mathrm{D}$ case. Now the reader can easily imagine what the authors would like to define. For notational simplicity, we put

$$
\text { " } n:=n-2, \quad \text { ' } n:=n-1, \quad n^{\prime}:=n+1, \quad n^{\prime \prime}:=n+2 .
$$

On the real $\left(t_{1}, \ldots, t_{n}\right)$-space, we fix $t_{0}<t_{n^{\prime}}<t_{n^{\prime \prime}}<\cdots$ and name the domains

$$
D_{0 \tilde{1} \cdots \tilde{n} n^{\prime} \ldots}:=\left\{\left(t_{1}, \ldots, t_{n}\right) \in \boldsymbol{R}^{n} \mid t_{0}<t_{1}<\cdots<t_{n}<t_{n^{\prime}}\right\}, \ldots .
$$

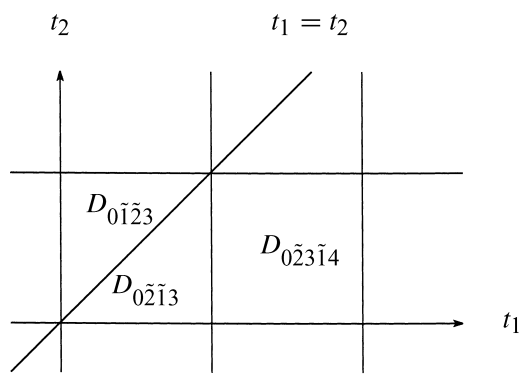

$$
t_{1}=t_{2} \quad t_{1}=t_{3}
$$

FIGURE 2. Coding the 2-domains. 
$\left(\tilde{1}, \ldots, \tilde{n}\right.$ indicate that $t_{1}, \ldots, t_{n}$ are variables, i.e., not fixed.) We array these intervals inductively as

$$
\begin{gathered}
D_{0 n^{\prime} n^{\prime \prime} \ldots}^{1 \cdots n}:={ }^{t}\left(D_{0 \tilde{n} n^{\prime} \ldots}^{1 \cdots{ }^{\prime} n}, D_{0 n^{\prime} \tilde{n} n^{\prime \prime} \ldots}^{1 \cdots \prime}, D_{0 n^{\prime} n^{\prime \prime} \tilde{n} \ldots}^{1 \cdots \prime}, \ldots\right), \ldots \\
\vdots \\
D_{0 \tilde{2} \cdots \tilde{n} n^{\prime} \ldots}^{1}:={ }^{t}\left(D_{0 \tilde{1} \tilde{2} \cdots \tilde{n} n^{\prime}}, D_{0 \tilde{2} \tilde{1} \cdots \tilde{n} n^{\prime}}, D_{0 \tilde{2} \tilde{3} \tilde{1} \cdots \tilde{n} n^{\prime}}, \ldots, D_{0 \tilde{2} \tilde{3} \cdots \tilde{n} \tilde{1} n^{\prime}}, \ldots\right), \ldots .
\end{gathered}
$$

In this subsection, the numerals with tildes among the sub-indices of $D$ indicate that the corresponding points are free (not fixed). When it is clear which points are fixed and which points are free, we often omit the tildes, later.

1.5 Standard loading. For complex constants $a_{i j} \in \boldsymbol{C}-\boldsymbol{Z}$, we consider the multivalued fuction

$$
u:=\prod_{i=1}^{n}\left(t_{i}-t_{0}\right)^{\alpha_{i 0}} \prod_{(i<) j}\left(t_{i}-t_{j}\right)^{a_{i j}},
$$

and the local system $\mathcal{L}$ defined by $u$. We load each domain $D$ with the function

$$
u:=\prod_{i=1}^{n}\left\{\varepsilon_{i 0}^{D}\left(t_{i}-t_{0}\right)\right\}^{\alpha_{i 0}} \prod_{(i<) j}\left\{\varepsilon_{i j}^{D}\left(t_{i}-t_{j}\right)\right\}^{a_{i j}},
$$

where $\varepsilon_{i 0}^{D}, \varepsilon_{i j}^{D}= \pm 1$ are so chosen that $\varepsilon_{i 0}^{D}\left(t_{i}-t_{0}\right)$ and $\varepsilon_{i j}^{D}\left(t_{i}-t_{j}\right)$ are positive on the domain $D$ (arguments of positve numbers are 0 ), and regard the domain as a loaded cycle. This loading is said to be standard. A standardly loaded cycle will often be called just by the name of the domain which supports the cycle.

1.6 Notation of the exponents.

$$
\begin{gathered}
a_{i j}=a_{j i}, \quad r_{i j}:=\exp \pi \sqrt{-1} a_{i j}, \\
r_{i j k}:=r_{i j} \cdot r_{j k} \cdot r_{k i}, \quad r_{i j \cdots k}:=\prod_{\{p, q\} \subset\{i, j, \ldots, k\}} r_{p q}, \\
c_{i j}:=r_{i j}^{2}, \quad c_{i j \cdots k}:=r_{i j \cdots k}^{2}, \\
d_{i j}:=c_{i j}-1, \quad d_{i j k}:=c_{i j k}-1, \ldots, \quad d_{i j \cdot p q}:=c_{i j} \cdot c_{p q}-1, \ldots .
\end{gathered}
$$

2. The half-turn formulae. Let $t_{a}<t_{b}$ be adjacent points in the sequence $t_{0}<$ $t_{n+1}<\cdots$. The two points $t_{a}$ and $t_{b}$ were fixed when we defined the $n$-dimensional domains in $\S 1$ such as $D_{0 \tilde{1} \tilde{2} \cdots \tilde{n} n^{\prime}}, D_{0 \tilde{2} \tilde{1} \cdots \tilde{n} n^{\prime}}, \ldots$. In this section we move $t_{b}$ in the complex plane in the counterclockwise direction around $t_{a}$. Accordingly the domains deform, and so do the loaded cycles. We describe the happening when the travel of $t_{b}$ is halfway completed, and $t_{a}$ and $t_{b}$ have exchanged positions. We assume that the resulting interval $\left[t_{b}, t_{a}\right]$ contains no other points in $\left\{t_{0}, t_{n+1}, \ldots\right\}$. Each of the (standardly loaded) cycles with respect to the sequence

$$
t_{0}<t_{n+1}<\cdots<t_{a}<t_{b}<\cdots
$$


is transformed into a linear combination of the (standardly loaded) cycles with respect to the sequence

$$
t_{0}<t_{n+1}<\cdots<t_{b}<t_{a}<\cdots
$$

This transformation is called the (positive) half-turn $H(a b)$ with respect to the points $t_{a}<t_{b}$. If $t_{b}$ continues traveling back to the original position, by applying the half-turn transformation (do not forget to exchange the corresponding exponents) again, we get the full-turn transformation $F(a b)$.

$2.11 \mathrm{D}$ case. Let $t_{a}<t_{b}$ be adjacent points in the sequence $t_{0}<t_{2}<t_{3}<\cdots$. The half-turn $H(a b)$ with respect to the points $t_{a}<t_{b}$ transforms the cycles as

$$
\begin{aligned}
& D_{1 a b} \rightarrow r_{a b}\left(D_{1 b a}+r_{1 b} D_{b 1 a}\right), \\
& D_{a 1 b} \rightarrow-r_{a 1 b} D_{b 1 a}, \\
& D_{a b 1} \rightarrow r_{a b}\left(D_{b a 1}+r_{a 1} D_{b 1 a}\right),
\end{aligned}
$$

other intervals $D \ldots a b \ldots$ being sent to $r_{a b} D \ldots b a \ldots$ (See Figure 3.) In this subsection, the tilde on the letter 1 is omitted.

$2.22 \mathrm{D}$ case. Let $t_{a}<t_{b}$ be adjacent points in the sequence $t_{0}<t_{3}<t_{4}<\cdots$. The half-turn $H(a b)$ with respect to the points $t_{a}<t_{b}$ transforms the cycles as

$$
\begin{aligned}
& D_{12 a b} \rightarrow r_{a b}\left(D_{12 b a}+r_{2 b} D_{1 b 2 a}+r_{2 b} r_{1 b} D_{b 12 a}\right), \\
& D_{1 a 2 b} \rightarrow-r_{a 2 b}\left(D_{1 b 2 a}+r_{1 b} D_{b 12 a}+r_{1 b} r_{12} D_{b 21 a}\right), \\
& D_{1 a b 2} \rightarrow r_{a b}\left(D_{1 b a 2}+r_{1 b} D_{b 1 a 2}+r_{a 2} D_{1 b 2 a}+r_{1 b} r_{a 2} D_{b 12 a}+r_{1 b} r_{a 2} r_{12} D_{b 21 a}\right), \\
& D_{a 12 b} \rightarrow r_{a 12 b} D_{b 21 a}, \\
& D_{a 1 b 2} \rightarrow-r_{a 1 b}\left(D_{b 1 a 2}+r_{a 2} D_{b 12 a}+r_{a 2} r_{12} D_{b 21 a}\right), \\
& D_{a b 12} \rightarrow r_{a b}\left(D_{b a 12}+r_{a 1} D_{b 1 a 2}+r_{a 1} r_{a 2} D_{b 12} a\right) .
\end{aligned}
$$

(See Figure 4.) If 1 or 2 is away from $a$ and $b$, then the move reduces to the $1 D$-case. In this subsection, the tildes on the letters 1 and 2 are omitted.

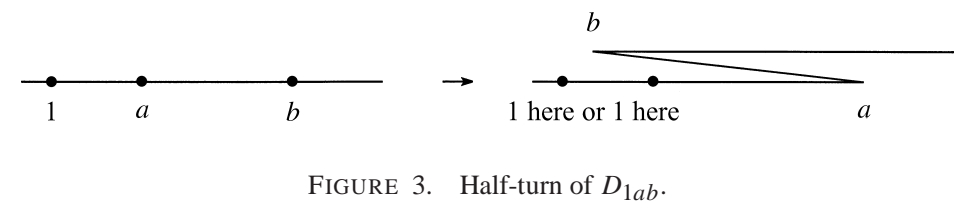




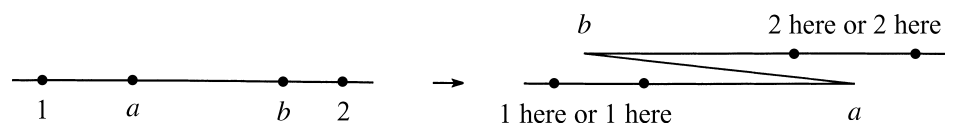

FIGURE 4. Half-turn of $D_{1 a b 2}$.

$2.33 \mathrm{D}$ case. Let $t_{a}<t_{b}$ be adjacent points in the sequence $t_{0}<t_{4}<t_{5}<\cdots$. The half-turn $H(a b)$ with respect to the points $t_{a}<t_{b}$ transforms the cycles as

$$
\begin{aligned}
& D_{123 a b} \rightarrow r_{a b}\left(D_{123 b a}+r_{3 b} D_{12 b 3 a}+r_{3 b} r_{2 b} D_{1 b 23 a}+r_{3 b} r_{2 b} r_{1 b} D_{b 123 a}\right), \\
& D_{12 a 3 b} \rightarrow-r_{a 3 b}\left(D_{12 b 3 a}+r_{2 b} D_{1 b 23 a}+r_{2 b} r_{23} D_{1 b 32 a}+r_{2 b} r_{1 b} D_{b 123 a}+r_{2 b} r_{1 b} r_{23} D_{b 132 a}\right. \\
& \left.+r_{2 b} r_{1 b} r_{23} r_{13} D_{b 312 a}\right) \text {, } \\
& D_{12 a b 3} \rightarrow r_{a b}\left(D_{12 b a 3}+r_{2 b} D_{1 b 2 a 3}+r_{2 b} r_{3 a} D_{1 b 23 a}+r_{2 b} r_{3 a} r_{32} D_{1 b 32 a}+r_{2 b} r_{1 b} D_{b 12 a 3}\right. \\
& \left.+r_{2 b} r_{1 b} r_{3 a} D_{b 123 a}+r_{2 b} r_{1 b} r_{3 a} r_{32} D_{b 132 a}+r_{2 b} r_{1 b} r_{3 a} r_{32} r_{31} D_{b 312 a}\right) \text {, } \\
& D_{1 a 23 b} \rightarrow r_{a 23 b}\left(D_{1 b 23 a}+r_{1 b} D_{b 123 a}+r_{1 b} r_{12} D_{b 213 a}+r_{1 b} r_{12} r_{31} D_{b 231 a}\right), \\
& D_{1 a 2 b 3} \rightarrow-r_{a 2 b}\left(D_{1 b 2 a 3}+r_{1 b} D_{b 12 a 3}+r_{a 3} D_{1 b 23 a}\right. \\
& +r_{1 b} r_{a 3} \sum_{\sigma} D_{b 1^{\sigma} 2^{\sigma} 3^{\sigma} a}(\sigma: \text { permutations of } 123) \text {, } \\
& D_{a 123 b} \rightarrow-r_{a 123 b} D_{b 321 a} \\
& D_{a 12 b 3}, \quad D_{a 1 b 23}, \quad D_{a b 123} \text { : as above. }
\end{aligned}
$$

If 1,2 or 3 is away from $a, b$ and $c$, then the move reduces to the 2D-case. In this subsection, the tildes on the letters 1,2 and 3 are omitted.

$2.4 n \mathrm{D}$ case. By the examples above, we hope that the reader can imagine what will happen in high-dimensional cases. Since the situation is fairly complicated, we state the action of half-turn properly.

A shuffle of the three ordered sets

$$
X=\left\{\ldots, x_{i}, x_{i+1}, \ldots\right\}, \quad Y=\left\{\ldots, y_{j}, y_{j+1}, \ldots\right\}, \quad Z=\left\{\ldots, z_{k}, z_{k+1}, \ldots\right\}
$$

is a permutaion of the elements of $X \cup Y \cup Z$ such that the order of the elements of $X$, the order of the elements of $Y$, and the order of the elements of $Z$ are preserved. The set of such shuffles is denoted by $S(X, Y, Z)$. For example, if

$$
X=\left\{x_{1}, x_{2}\right\}, \quad Y=\left\{y_{1}, y_{2}\right\}, \quad Z=\emptyset,
$$

then

$S(X, Y, Z)=\left\{x_{1} x_{2} y_{1} y_{2}, x_{1} y_{1} x_{2} y_{2}, x_{1} y_{1} y_{2} x_{2}, y_{1} x_{1} x_{2} y_{2}, y_{1} x_{1} y_{2} x_{2}, y_{1} y_{2} x_{1} x_{2}\right\}$.

For two members $x$ and $y$ of a shuffle $s$, if $x$ is on the left side of $y$, we denote ' $x \prec y$ in $s$ '. Let $Y^{-1}$ be the order reversed set of $Y$, that is,

$$
Y^{-1}=\left\{\ldots, y_{j+1}, y_{j}, \ldots\right\} .
$$




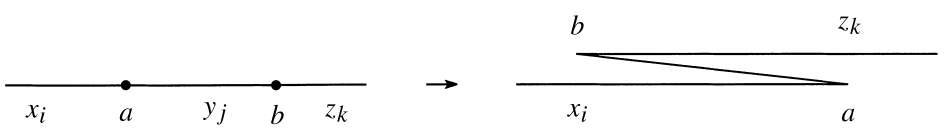

FigURE 5. Half-turn $H(a, b)$.

For a shuffle $s \in S\left(X, Y^{-1}, Z\right)$, define $\varepsilon_{S}(\cdot, \cdot)$ as

$$
\varepsilon_{s}\left(x_{i}, y_{j}\right):=\left\{\begin{array}{ll}
0 & \text { if } x_{i} \prec y_{j} \text { in } s, \\
1 & \text { if } x_{i} \succ y_{j} \text { in } s,
\end{array} \quad \varepsilon_{s}\left(y_{j}, z_{k}\right):= \begin{cases}0 & \text { if } y_{j} \prec z_{k} \text { in } s, \\
1 & \text { if } y_{j} \succ z_{k} \text { in } s,\end{cases}\right.
$$

and

$$
\varepsilon_{s}\left(x_{i}, z_{k}\right):= \begin{cases}0 & \text { if } x_{i} \prec z_{k} \text { in } s \\ 1 & \text { if } x_{i} \succ z_{k} \text { in } s .\end{cases}
$$

Consider an $n$-dimensional domain $D_{* * *}$ defined in $\$ 1.4$. Recall that it is coded by an arrangement of $n$ moving points $t_{1}, \ldots, t_{n}$ in the real line divided by fixed ones $t_{0}<$ $t_{n+1}<\cdots$. Let $t_{a}<t_{b}$ be adjacent points in the sequence $t_{0}<t_{n+1}<\cdots$, and $t_{a^{\prime}}$ be the left-adjacent point (if any) to $t_{a}$, and $t_{b^{\prime}}$ the right-adjacent point to $t_{b}$. Let $t_{X}, t_{Y^{\prime}}$ and $t_{Z}$ be the ordered subsets of $\left\{t_{0}, \ldots, t_{n}\right\}$ situated in the interval $\left[t_{a^{\prime}}, t_{a}\right],\left[t_{a}, t_{b}\right]$ and $\left[t_{b}, t_{b^{\prime}}\right]$, respectively. The indices of the points in $t_{X}, t_{Y^{\prime}}$ and $t_{Z}$ define ordered subsets $X, Y^{\prime}$ and $Z$ of $\{0, \ldots, n\}$; put $Y=\left\{a, Y^{\prime}, b\right\}$. Thus the domain $D_{* * *}$ has the coding $D \ldots X Y Z \ldots$. Now we are ready to state the move of the half-turn.

PROPOSITION 1. Let a be the left extreme element of $Y$, and $b$ the right extreme. Suppose that $X \cup(Y-\{a, b\}) \cup Z \subset\{1, \ldots, n\}$. Then the half-turn $H(a b)$ with respect to the points $t_{a}<t_{b}$ transforms the cycles

$$
D \ldots X Y Z \ldots=D_{\ldots x_{i} x_{i+1} \ldots a \ldots y_{j} y_{j+1} \ldots b \ldots z_{k} z_{k+1} \ldots}
$$

into

where

$$
(-)^{\# Y} r_{Y} \sum_{s \in S\left(X, Y^{-1}, Z\right)} r(s) D \ldots s \cdots,
$$

$$
r(s)=\prod_{i, j, k} r_{x_{i} y_{j}}^{\varepsilon_{s}\left(x_{i}, y_{j}\right)} r_{y_{j} z_{k}}^{\varepsilon_{s}\left(y_{j}, z_{k}\right)} r_{x_{i} z_{k}}^{\varepsilon_{s}\left(x_{i}, z_{k}\right)}, \quad r_{Y}=\prod_{\{p, q\} \subset Y} r_{p q} .
$$

Proof. See Figure 5. Remember that $x_{i}$ move on the left-side of $a, y_{j}$ between $a$ and $b$, and $z_{k}$ on the right-side of $b$. After the half-turn of $b$ around $a$, let us regard

$$
\ldots x_{i} x_{i+1} \ldots b \ldots y_{j+1} y_{j} \ldots a \ldots z_{k} z_{k+1} \ldots
$$

as the ground state. Starting from this ground state, the very right one among $\left\{x_{i}\right\}$ can pass $b$ and the $y_{j}$ 's, the very left one among $\left\{z_{k}\right\}$ can pass $a$ and the $y_{j}$ 's; then the next right one among $\left\{x_{i}\right\}$ and so on, and we get shuffles of $X, Y^{-1}$ and $Z$. Each time when $x_{i}$ passes $y_{j}$, the argument of $t_{x_{i}}-t_{y_{j}}$ increases by $\pi$, so $r_{x_{i} y_{j}}$ is multiplied. This is also the case when $x_{i}$ passes $z_{k}$, and $z_{k}$ passes $y_{j}$. 
Since the moves of $y_{j}$ are reversed, for the orientation reason, we have the overall factor $(-)^{\# Y}$. The other overall factor $r_{Y}$ comes from the factors

$$
\prod_{p<q}\left(t_{y_{p}}-t_{y_{q}}\right)^{\alpha_{y_{p} y_{q}}}
$$

of $u$; note that the half-turn increases the argument of every factor by $\pi$.

3. Intersection numbers. Consider for each positive integer $n$ and a sequence of mutually distinct numbers

$$
t_{0}, \quad t_{n+1}, \quad t_{n+2}, \ldots,
$$

a domain in $C^{n}$ defined by

$$
X_{t_{0}, t_{n+1}, \ldots}^{1 \cdots n}:=\left\{\left(t_{1}, \ldots, t_{n}\right) \in C^{n} \mid t_{i} \neq t_{j}, 1 \leq i \leq n, 0 \leq j \neq i\right\} .
$$

The local system $\mathcal{L}$ (of rank 1 ) is defined by the function

$$
u:=\prod_{i=1}^{n}\left(t_{i}-t_{0}\right)^{\alpha_{i 0}} \prod_{(i<) j}\left(t_{i}-t_{j}\right)^{a_{i j}}
$$

on $X_{t_{0}, t_{n+1}}^{1 \cdots n}$. Via the projection

$$
\pi: X_{t_{0}, t_{n+1}, \ldots}^{1 \cdots n} \ni\left(t_{1}, \ldots, t_{n}\right) \longmapsto t_{n} \in X_{t_{0}, t_{n+1}, \ldots}^{n}:=\left\{t_{n} \mid t_{n} \neq t_{0}, t_{n+1}, \ldots\right\},
$$

the space $X_{t_{0}, t_{n+1}}^{1 \cdots n}$ can be regarded as a fibre bundle over the 1-dimensional space $X_{t_{0}, t_{n+1}, \ldots}^{n}$ with fibre

$$
\pi^{-1}\left(t_{n}\right)=X_{t_{0}, t_{n}, \ldots}^{1 \cdots(n-1)} .
$$

As explained in $\S 5$, we have the isomorphism

$$
H_{n}\left(X_{t_{0}, t_{n+1}, \ldots}^{1 \cdots n}, \mathcal{L}\right) \cong H_{1}\left(X_{t_{0}, t_{n+1}, \ldots}^{n}, \mathcal{H}_{n-1}\right),
$$

where $\mathcal{H}_{n-1}$ is the locally constant sheaf of germs of locally flat sections of the bundle

$$
\bigcup_{t_{n} \in X_{t_{0}, t_{n+1}, \ldots}^{n}} H_{n-1}\left(X_{t_{0}, t_{n}, \ldots}^{1 \cdots(n-1)}, \iota^{*} \mathcal{L}\right),
$$

where $\iota: X_{t_{0}, t_{n}, \ldots}^{1 \cdots(n-1)} \rightarrow X_{t_{0}, t_{n+1}, \ldots}^{1 \cdots n}$ is the inclusion.

When $t_{0}<t_{n+1}<t_{n+2}<\cdots$, the set $D_{0(n+1) \cdots}^{1 \cdots n}$ of loaded cycles form a basis of $H_{n}^{\mathrm{lf}}\left(X_{t_{0}, t_{n+1}, \ldots,}^{1 \cdots n}, \mathcal{L}\right)$. Assume that the $(n-1)$-dimensional intersection numbers of the cycles in $D_{0 n \cdots}^{1 \cdots(n-1)}$ and their duals are already known. Then the intersection numbers of the cycles

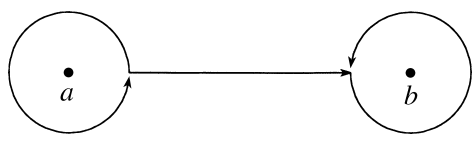

FIGURE 6. $\operatorname{reg} I_{a b}$. 
in $D_{0(n+1) \ldots}^{1 \ldots n}$ and their duals can be evaluated as follows. For adjacent points $t_{a}<t_{b}(a, b \in$ $\{0, n+1, \ldots\})$ in $t_{n}$-space, consider the interval $I_{a b}:=\left(t_{a}, t_{b}\right) \subset X_{t_{0}, t_{n+1}, \ldots}^{n}$ and load it with a section $\sigma$ of $\mathcal{H}_{n-1}^{\text {lf }}$ to be an element $I_{a b} \otimes \sigma$ of $H_{1}^{\text {lf }}\left(X_{t_{0}, t_{n+1}, \ldots}^{n}, \mathcal{H}_{n-1}^{\text {lf }}\right)$. We regularize the cycle $I_{a b} \otimes \sigma$ as

$$
\begin{aligned}
\operatorname{reg} I_{a b} \otimes \sigma:= & S(a ; a+\varepsilon) \otimes(F(a n)-\mathrm{id})^{-1} \sigma+(a+\varepsilon, b-\varepsilon) \otimes \sigma \\
& -S(b ; b-\varepsilon) \otimes(F(n b)-\mathrm{id})^{-1} \sigma,
\end{aligned}
$$

where $S(a ; a+\varepsilon)$ is a positively oriented circle with center $a$ and radius $\varepsilon$ starting and ending at $a+\varepsilon$ (see Figure 6). Also,

$$
F(n b): H_{n-1}^{\mathrm{lf}}\left(X_{t_{0}, \ldots, t_{a}, t_{n}, t_{b}, \ldots}^{1 \cdots(n-1)}, \iota^{*} \mathcal{L}\right) \rightarrow H_{n-1}^{\mathrm{lf}}\left(X_{t_{0}, \ldots, t_{a}, t_{n}, t_{b}, \ldots}^{1 \cdots(n-1)}, \iota^{*} \mathcal{L}\right)
$$

is the full-turn operator with respect to the move of the point $t_{n}$ along the circle $S(b ; b-\varepsilon)$.

For adjacent points $t_{c}<t_{d}$ in the $t_{n}$-space, consider the loaded cycle $I_{c d} \otimes \check{\sigma}$ with support on the interval $\left(t_{c}, t_{d}\right)$ and loaded with a section $\check{\sigma}$ of $\check{\mathcal{H}}_{n-1}^{\text {lf }}$ to be an element of $H_{1}^{\mathrm{lf}}\left(X_{t_{0}, t_{n+1}, \ldots}^{n}, \check{\mathcal{H}}_{n-1}^{\mathrm{lf}}\right)$. If $(a, b)=(c, d)$, then the intersection number $\left(I_{a b} \otimes \sigma\right) \bullet\left(I_{c d} \otimes \check{\sigma}\right)$ is given $(\$ 5)$ by

$$
\begin{aligned}
\left(\operatorname{reg} I_{a b} \otimes \sigma\right) \bullet\left(I_{c d} \otimes \check{\sigma}\right)= & -\left.\left\{(F(a n)-\mathrm{id})^{-1} \sigma\right\} \bullet \check{\sigma}\right|_{t_{n}=a+\varepsilon} \\
& -\left.\sigma \bullet \check{\sigma}\right|_{t_{n}=(a+b) / 2}-\left.\left\{(F(n b)-\mathrm{id})^{-1} \sigma\right\} \bullet \check{\sigma}\right|_{t_{n}=b-\varepsilon} ;
\end{aligned}
$$

recall that we assumed that the $(n-1)$-dimensional intersection numbers are already known. If $b=c$, then $\left(I_{a b} \otimes \sigma\right) \bullet\left(I_{c d} \otimes \check{\sigma}\right)$ is given by

$$
\left.\left\{(F(n b)-\mathrm{id})^{-1} H(n b) \sigma\right\} \bullet \check{\sigma}\right|_{t_{n}=b+\varepsilon},
$$

where

$$
H(n b): H_{n-1}^{\mathrm{lf}}\left(X_{t_{0}, \ldots, t_{a}, t_{n}, t_{b}, \ldots}^{1 \cdots(n-1)}, \iota^{*} \mathcal{L}\right) \rightarrow H_{n-1}^{\mathrm{lf}}\left(X_{\left.t_{0}, \ldots, t_{a}, t_{b}, t_{n}, \ldots, \iota^{*} \mathcal{L}\right)}^{1 \cdots(n-1)}\right.
$$

is the half-turn operator with respect to the move of the point $t_{n}$ along the half of the circle $S(b, b-\varepsilon)$ (note that $F(n b)=H(n b) H(b n))$; if $d=a$, then $\left(I_{a b} \otimes \sigma\right) \bullet\left(I_{c d} \otimes \check{\sigma}\right)$ is given by

otherwise 0 .

$$
\left.\left\{(F(a n)-\mathrm{id})^{-1} H(a n)\right\} \sigma \bullet \check{\sigma}\right|_{t_{n}=a-\varepsilon},
$$

The following examples show the actual process. We represent the half-turn and full-turn operators $H(. \cdot)$ and $F(. \cdot)$ by matrices, which will be denoted by roman letters $\mathrm{H}(. \cdot)$ and $\mathrm{F}(. \cdot)$, respectively.

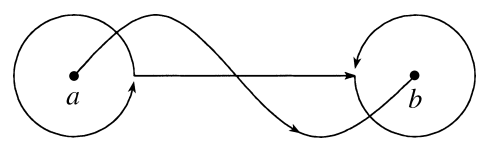

FIGURE 7. $\left(\operatorname{reg} I_{a b} \otimes \sigma\right) \bullet\left(I_{a b} \otimes \check{\sigma}\right)$. 


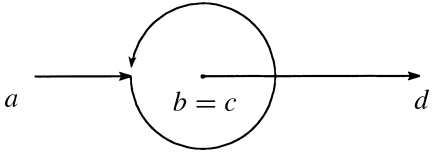

FIGURE 8. $\quad\left(\operatorname{reg} I_{a b} \otimes \sigma\right) \bullet\left(I_{b d} \otimes \check{\sigma}\right)$.

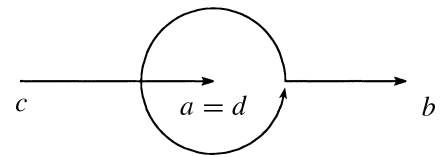

FIGURE 9. $\quad\left(\operatorname{reg} I_{a b} \otimes \sigma\right) \bullet\left(I_{c a} \otimes \check{\sigma}\right)$.

3.1 1D case. The half-turn $H(1 a)$ of $t_{1}$ around $t_{a}$ is represented by the scalar $\mathrm{H}(1 a)=$ $r_{1 a}$, and the full-turn $F(1 a)$ by the scalar $\mathrm{F}(1 a)=c_{1 a}=r_{1 a}^{2}$. For adjacent $t_{a}<t_{b}$, we have

$$
-\left(c_{a 1}-1\right)^{-1}-1-\left(c_{1 b}-1\right)^{-1}=-\frac{d_{a 1 \cdot 1 j}}{d_{a 1} d_{1 b}}, \quad\left(c_{1 b}-1\right)^{-1} r_{1 b}=\frac{r_{1 b}}{d_{1 b}} .
$$

The intersection matix is given as

$$
I_{02 \ldots}^{1}:=D_{02 \ldots}^{1} \bullet \check{D}_{02 \ldots}^{1}=\left(\begin{array}{cccc}
-\frac{d_{01 \cdot 12}}{d_{01} d_{12}} & \frac{r_{12}}{d_{12}} & 0 & \ldots \\
\frac{r_{21}}{d_{21}} & -\frac{d_{21 \cdot 13}}{d_{21} d_{13}} & \frac{r_{13}}{d_{13}} & 0 \\
0 & \frac{r_{31}}{d_{31}} & -\frac{d_{31 \cdot 14}}{d_{31} d_{14}} & \ddots \\
\vdots & 0 & \ddots & \ddots
\end{array}\right)
$$

3.2 2D case. For adjacent $t_{a}<t_{2}<t_{b}$, let us represent the half-turns and the fullturns of $t_{2}$ around $t_{a}$ and $t_{b}$ by matrices with respect to the column vectors $D_{\cdots a 2 b \ldots}^{1}$ and $D_{\ldots 2 a b \ldots}^{1}$ as

$$
\begin{aligned}
& H(a 2) D_{\cdots a 2 b \cdots}^{1}=\mathrm{H}(a 2) D_{\cdots 2 a b \cdots}^{1}, \quad F(a 2) D_{\cdots a 2 b \cdots}^{1}=\mathrm{F}(a 2) D_{\ldots a 2 b \cdots}^{1}, \\
& H(2 b) D_{\cdots a 2 b \cdots}^{1}=\mathrm{H}(2 b) D_{\cdots a b 2 \cdots}^{1}, \quad F(2 b) D_{\cdots a 2 b \cdots}^{1}=\mathrm{F}(2 b) D_{\cdots a 2 b \cdots}^{1} .
\end{aligned}
$$

Our convention on the product of operators is from the left to the right; for example, $F(a 2)=$ $H(a 2) H(2 a)$ operates $H(a 2)$ first and then $H(2 a)$; so we have $\mathrm{F}(a 2)=\mathrm{H}(a 2) \mathrm{H}(2 a)$. Put

$$
\begin{aligned}
& \mathrm{F}(a 2 b):=-(\mathrm{F}(a 2)-\mathrm{id})^{-1}-\mathrm{id}-(\mathrm{F}(2 b)-\mathrm{id})^{-1}, \\
& \mathrm{G}(2 b):=(\mathrm{F}(2 b)-\mathrm{id})^{-1} \mathrm{H}(2 b) .
\end{aligned}
$$


The 2D-intersection matrix is given as

$$
\begin{aligned}
I_{03 \ldots}^{12}= & D_{03 \ldots}^{12} \bullet \check{D}_{03 \ldots}^{12}=\left(\begin{array}{c}
D_{0 \tilde{2} 3 \ldots}^{1} \\
D_{03 \tilde{2} 4 \ldots}^{1} \\
\vdots
\end{array}\right) \bullet\left({ }^{t} \check{D}_{02 \tilde{3} 3 \ldots}^{1},{ }^{t} \check{D}_{03 \tilde{2} 4 \ldots}^{1} \ldots\right) \\
& =\left(\begin{array}{cccc}
\mathrm{F}(023) & \mathrm{G}(23) & 0 & \cdots \\
\mathrm{G}(32) & \mathrm{F}(324) & \mathrm{G}(24) & 0 \\
0 & \mathrm{G}(42) & \mathrm{F}(425) & \ddots \\
\vdots & 0 & \ddots & \ddots
\end{array}\right)\left(\begin{array}{cccc}
I_{023 \ldots}^{1} & 0 & 0 & \cdots \\
0 & I_{0324 \ldots}^{1} & 0 & 0 \\
0 & 0 & I_{03425 \ldots}^{1} & \ddots \\
\vdots & 0 & \ddots & \ddots
\end{array}\right),
\end{aligned}
$$

where $I_{* *}^{1}$ are already evaluated 1D-intersection matrices:

$$
I_{023 \ldots}^{1}=\left(\begin{array}{c}
D_{0 \tilde{1} 2 \ldots} \\
D_{02 \tilde{1} 3 \ldots} \\
\vdots
\end{array}\right) \bullet\left({ }^{t} \check{D}_{0 \tilde{1} 2 \ldots}, \ldots\right), \quad I_{0324 \ldots}^{1}=\left(\begin{array}{c}
D_{0 \tilde{1} 324 \ldots} \\
D_{03 \tilde{1} 24 \ldots} \\
\vdots
\end{array}\right) \bullet\left({ }^{t} \check{D}_{0 \tilde{1} 324 \ldots}, \ldots\right), \ldots
$$

3.3 3D case. For adjacent $t_{a}<t_{3}<t_{b}$, let us represent the half-turns and the fullturns of $t_{3}$ around $t_{a}$ and $t_{b}$ by matrices with respect to the column vectors $D_{\cdots a 3 b \ldots}^{12}$ and $D_{\ldots 3 a b \cdots}^{12}$ :

$$
\begin{aligned}
& H(a 3) D_{\cdots a 3 b \cdots}^{12}=\mathrm{H}(a 3) D_{\cdots 3 a b \cdots}^{12}, \quad F(a 3) D_{\cdots a 3 b \cdots}^{12}=\mathrm{F}(a 3) D_{\cdots a 3 b \cdots}^{12}, \\
& H(3 b) D_{\cdots a 3 b \cdots}^{12}=\mathrm{H}(3 b) D_{\cdots a b 3 \cdots}^{12}, \quad F(3 b) D_{\cdots a 3 b \cdots}^{12}=\mathrm{F}(3 b) D_{\cdots a 3 b \cdots}^{12}
\end{aligned}
$$

Put

$$
\begin{aligned}
\mathrm{F}(a 3 b) & :=-(\mathrm{F}(a 3)-\mathrm{id})^{-1}-\mathrm{id}-(\mathrm{F}(3 b)-\mathrm{id})^{-1}, \\
\mathrm{G}(3 b) & :=(\mathrm{F}(3 b)-\mathrm{id})^{-1} H(3 b) .
\end{aligned}
$$

The 3D-intersection matrix is given as

$$
\begin{aligned}
I_{04 \ldots}^{123}: & =D_{04 \ldots}^{123} \bullet \check{D}_{04 \ldots}^{123}=\left(\begin{array}{c}
D_{0 \tilde{3} 4 \ldots}^{12} \\
D_{04 \tilde{3} 5 \ldots}^{12} \\
\vdots
\end{array}\right) \bullet\left({ }^{t} \check{D}_{0 \tilde{3} 4 \ldots}^{12},{ }^{t} \check{D}_{04 \tilde{3} 5 \ldots}^{12}, \ldots\right) \\
& =\left(\begin{array}{cccc}
\mathrm{F}(034) & \mathrm{G}(34) & 0 & \cdots \\
\mathrm{G}(43) & \mathrm{F}(435) & \mathrm{G}(35) & 0 \\
0 & \mathrm{G}(53) & \mathrm{F}(536) & \ddots \\
\vdots & 0 & \ddots & \ddots
\end{array}\right)\left(\begin{array}{cccc}
I_{034 \ldots}^{12} & 0 & 0 & \cdots \\
0 & I_{0435 \ldots}^{12} & 0 & 0 \\
0 & 0 & I_{04536}^{12} \ldots & \ddots \\
\vdots & 0 & \ddots & \ddots
\end{array}\right),
\end{aligned}
$$

where $I_{* *}^{12}$ are already evaluated 2D-intersection matrices. 
3.4 $n \mathrm{D}$ case. Now the reader can easily imagine what will happen in high-dimensional cases. For adjacent $t_{a}<t_{n}<t_{b}$, let us represent the half-turns and the full-turns of $t_{n}$ around $t_{a}$ and $t_{b}$ by matrices with respect to the column vectors $D_{\cdots a n b \cdots}^{1 \cdots n^{\prime}}$ and $D_{\cdots n a b \cdots}^{1 \cdots n^{\prime}}$ :

$$
\begin{aligned}
& H(a n) D_{\cdots a n b \cdots}^{1 \cdots n^{\prime}}=\mathrm{H}(a n) D_{\cdots n a b \cdots}^{1 \cdots n^{\prime}}, \quad F(a n) D_{\cdots a n b \cdots}^{1 \cdots n^{\prime}}=\mathrm{F}(a n) D_{\cdots a n b \cdots}^{1 \cdots n^{\prime}}, \\
& H(n b) D_{\cdots a n b \cdots}^{1 \cdots n^{\prime}}=\mathrm{H}(n b) D_{\cdots a b n \cdots}^{1 \cdots n^{\prime}}, \quad F(n b) D_{\cdots a n b \cdots}^{1 \cdots n^{\prime}}=\mathrm{F}(n b) D_{\cdots a n b \cdots}^{1 \cdots n^{\prime}},
\end{aligned}
$$

Put

$$
\begin{aligned}
& \mathrm{F}(a n b):=-(\mathrm{F}(a n)-\mathrm{id})^{-1}-\mathrm{id}-(\mathrm{F}(n b)-\mathrm{id})^{-1}, \\
& \mathrm{G}(4 b):=(\mathrm{F}(4 b)-\mathrm{id})^{-1} \mathrm{H}(4 b) .
\end{aligned}
$$

PROPOSITION 2. The $n D$-intersection matrix is given as

$$
\begin{aligned}
& I_{0 n^{\prime} \cdots}^{12 \cdots n}:=D_{0 n^{\prime} \cdots n}^{12 \ldots n} \bullet \check{D}_{0 n^{\prime} \cdots}^{12 \ldots n}=\left(\begin{array}{c}
D_{0 \tilde{n} n^{\prime} \ldots}^{1 \ldots{ }^{\prime} n} \\
D_{0 n^{\prime} \tilde{n} n^{\prime \prime} \ldots}^{1 \ldots{ }^{\prime}} \\
\vdots
\end{array}\right) \bullet\left({ }^{t} \check{D}_{0 \tilde{n} n^{\prime} \ldots}^{1 \ldots{ }^{\prime} n},{ }^{t} \check{D}_{\left.0 n^{\prime} \tilde{n} n^{\prime \prime} \ldots, \ldots\right)}^{1 \ldots{ }^{\prime} \ldots}\right. \\
& =\left(\begin{array}{cccc}
\mathrm{F}\left(0 n n^{\prime}\right) & \mathrm{G}\left(n n^{\prime}\right) & 0 & \cdots \\
\mathrm{G}\left(n^{\prime} n\right) & \mathrm{F}\left(n^{\prime} n n^{\prime \prime}\right) & \mathrm{G}\left(n n^{\prime \prime}\right) & 0 \\
0 & \mathrm{G}\left(n^{\prime \prime} n\right) & \mathrm{F}\left(n^{\prime \prime} n n^{\prime \prime \prime}\right) & \ddots \\
\vdots & 0 & \ddots & \ddots
\end{array}\right)\left(\begin{array}{cccc}
I_{0 n n^{\prime} \cdots}^{1 \ldots{ }^{\prime}} & 0 & 0 & \cdots \\
0 & I_{0 n^{\prime} n n^{\prime \prime} \ldots}^{1 \ldots \prime} & 0 & 0 \\
0 & 0 & I_{0 n^{\prime} n^{\prime \prime} n n^{\prime \prime \prime} \ldots}^{1 \ldots} \cdot \\
\vdots & 0 & \ddots & \ddots
\end{array}\right),
\end{aligned}
$$

where $I_{* *}^{1 \ldots ' n}$ are already evaluated $(n-1) D$-intersection matrices.

4. An application to the Selberg integral. To show that our inductive method can serve as practical use, we derive the result obtained in [MiY1] and [MiY2] as applications of our method in 1,2 and 3-dimensional cases.

So far, we considered infinitely many $t_{i}$ 's, and the exponents $\alpha_{i j}$ were independent. Now we work in $\left(t_{1}, \ldots, t_{n}\right)$-space with the three kinds of hyperplanes

$$
\left(t_{0}=\right) 0=t_{j}, \quad t_{j}=1\left(=t_{n+1}\right), \quad t_{i}=t_{j} \quad(1 \leq i, j \leq n)
$$

with respective exponents

$$
c_{01}=\cdots=c_{0 n}=a, \quad c_{1(n+1)}=\cdots=c_{n(n+1)}=b, \quad r_{i j}=g \quad(1 \leq i, j \leq n) .
$$

The integral in question is the Selberg integral

$$
\int u \frac{d t_{1} \wedge \cdots \wedge d t_{n}}{\prod_{i=1}^{n} t_{i}\left(1-t_{i}\right)}, \quad u=\prod_{i=1}^{n} t_{i}^{a}\left(1-t_{i}\right)^{b} \prod_{1 \leq i<j \leq n}\left|t_{i}-t_{j}\right|^{2 g} .
$$

The symmetric group acts on the coordinates $t_{1}, \ldots, t_{n}$ of

$$
X^{n}=\left\{\left(t_{1}, \ldots, t_{n}\right) \in \boldsymbol{C}^{n} \mid t_{i} \neq 0,1, t_{j}(i \neq j)\right\} .
$$


So it acts also on the homology group $H_{n}^{\text {lf }}\left(X^{n}, \mathcal{L}\right)$. Its $S_{n}$-invariant subspace is 1-dimensional, and is generated by

$$
C_{n}:=\sum_{\sigma \in S_{n}} D_{01^{\sigma}} \widetilde{2}^{\sigma} \ldots \widetilde{n^{\sigma}}(n+1)
$$

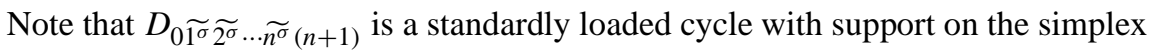

$$
\left\{\left(t_{1}, \ldots, t_{n}\right) \in \boldsymbol{R}^{n} \mid 0<t_{1} \sigma<\cdots<t_{n^{\sigma}}<1\right\} .
$$

In this section we evaluate the $n \mathrm{D}$-intersection number $J_{n}:=C_{n} \bullet \check{C}_{n}$ for $n=1,2,3$.

4.1 1D case.

$$
J_{1}=D_{0 \tilde{1} 2} \bullet \check{D}_{0 \tilde{1} 2}=-\frac{d_{01 \cdot 12}}{d_{01} d_{12}}=\frac{1-a b}{(1-a)(1-b)} .
$$

4.2 2D case. We evaluate $J_{2}=\left(D_{0 \tilde{1} \tilde{2} 3}+D_{0 \tilde{2} \tilde{1} 3}\right) \bullet\left(\check{D}_{0 \tilde{1} \tilde{2} 3}+\check{D}_{0 \tilde{2} \tilde{1} 3}\right)$. Recall that these intersection numbers are computed as

$$
\begin{aligned}
I_{03}^{12} & :=D_{03}^{12} \bullet \check{D}_{03}^{12}=D_{0 \tilde{2} 3}^{1} \bullet{ }^{t} \check{D}_{0 \tilde{2} 3}^{1}=\left(\begin{array}{c}
D_{0 \tilde{1} \tilde{2} 3} \\
D_{0 \tilde{1} \tilde{1} 3}
\end{array}\right) \bullet\left(\check{D}_{0 \tilde{1} \tilde{2} 3}, \check{D}_{0 \tilde{2} \tilde{1} 3}\right) \\
& =\mathrm{F}(023) I_{023}^{1},
\end{aligned}
$$

where

$$
I_{023}^{1}:=D_{023}^{1} \bullet{ }^{t} \check{D}_{023}^{1}=\left(\begin{array}{c}
D_{0 \tilde{1} 23} \\
D_{02 \tilde{1} 3}
\end{array}\right) \bullet\left(\check{D}_{0 \tilde{1} 23}, \check{D}_{02 \tilde{1} 3}\right)=-\left(\begin{array}{cc}
\frac{d_{01 \cdot 12}}{d_{01} d_{12}} & -\frac{r_{12}}{d_{12}} \\
-\frac{r_{21}}{d_{21}} & \frac{d_{21 \cdot 13}}{d_{21} d_{13}}
\end{array}\right),
$$

and

$$
\mathrm{F}(02):=\mathrm{H}(02) \mathrm{H}(20)=\left(\begin{array}{cc}
-r_{012} & 0 \\
r_{02} r_{01} & r_{02}
\end{array}\right)\left(\begin{array}{cc}
-r_{012} & 0 \\
r_{20} r_{12} & r_{20}
\end{array}\right),
$$

$$
\mathrm{F}(023):=-(\mathrm{F}(02)-\mathrm{id})^{-1}-\mathrm{id}-(\mathrm{F}(23)-\mathrm{id})^{-1}
$$

$$
\begin{aligned}
& =-\left(\begin{array}{cc}
c_{012}-1 & 0 \\
-c_{02} r_{12} d_{01} & c_{02}-1
\end{array}\right)^{-1}-\mathrm{id}-\left(\begin{array}{cc}
c_{23}-1 & -c_{23} r_{12} d_{13} \\
0 & c_{123}-1
\end{array}\right)^{-1} \\
& =-\left(\begin{array}{cc}
\frac{1}{d_{012}}+1+\frac{1}{d_{23}} & \frac{c_{23} r_{12} d_{13}}{d_{23} d_{123}} \\
\frac{c_{02} r_{12} d_{01}}{d_{012} d_{02}} & \frac{1}{d_{02}}+1+\frac{1}{d_{123}}
\end{array}\right)=-\left(\begin{array}{cc}
\frac{d_{012 \cdot 23}}{d_{012} d_{23}} & \frac{c_{23} r_{12} d_{13}}{d_{23} d_{123}} \\
\frac{c_{02} r_{12} d_{01}}{d_{012} d_{02}} & \frac{d_{02 \cdot 123}}{d_{02} d_{123}}
\end{array}\right) .
\end{aligned}
$$

Now put

$$
c_{01}=c_{02}=a, \quad c_{13}=c_{23}=b, \quad r_{12}=g,
$$

and add the entries of

$$
\mathrm{F}(023)=\left(\begin{array}{cc}
\frac{1-a^{2} g^{2} b}{\left(1-a^{2} g^{2}\right)(1-b)} & \frac{g b}{1-g^{2} b^{2}} \\
\frac{a g}{1-a^{2} g^{2}} & \frac{1-a g^{2} b^{2}}{(1-a)\left(1-g^{2} b^{2}\right)}
\end{array}\right)
$$


vertically:

$$
\left(f_{1}, f_{2}\right):=\left(\frac{1-a g b}{(1-a g)(1-b)}, \frac{1-a g b}{(1-a)(1-g b)}\right),
$$

and add the entries of

$$
I_{023}^{1}=\left(\begin{array}{cc}
\frac{1-a g^{2}}{(1-a)\left(1-g^{2}\right)} & -\frac{g}{1-g^{2}} \\
-\frac{g}{1-g^{2}} & \frac{1-g^{2} b}{\left(1-g^{2}\right)(1-b)}
\end{array}\right)
$$

horizontally:

$$
{ }^{t}\left(g_{1}, g_{2}\right):=\left(\frac{a g+1}{(1-a)(g+1)}, \frac{g b+1}{(g+1)(1-b)}\right) .
$$

We thus have

$$
\begin{aligned}
J_{2} & =f_{1} g_{1}+f_{2} g_{2}=\frac{1-a g b}{(1-a)(1-b)(g+1)}\left(\frac{a g+1}{a g-1}+\frac{g b+1}{1-g b}\right) \\
& =\frac{2(1-a g b)\left(1-a g^{2} b\right)}{(1-a)(1-a g)(1-b)(1-g b)(g+1)},
\end{aligned}
$$

which agrees with the result in [MiY1].

$4.33 \mathrm{D}$ case. We evaluate $J_{3}:=C_{3} \bullet \check{C}_{3}$, where $C_{3}=\sum_{\sigma \in S_{3}} D_{0 \widetilde{1}^{\sigma} \widetilde{2}^{\sigma} \widetilde{3}^{\sigma} 4}$. Recall that these intersection numbers are computed as

$$
\begin{aligned}
I_{04}^{123}: & =D_{04}^{123} \bullet \check{D}_{04}^{123}=D_{0 \tilde{3} 4}^{12} \bullet{ }^{t} \check{D}_{0 \tilde{3} 4}^{12}=\left(\begin{array}{c}
D_{0 \tilde{2} \tilde{3} 4}^{1} \\
D_{0 \tilde{3} \tilde{2} 4}^{1}
\end{array}\right) \bullet\left({ }^{t} \check{D}_{0 \tilde{2} \tilde{3} 4}^{1},{ }^{t} \check{D}^{1}{ }_{0 \tilde{3} \tilde{2} 4}\right) \\
& =\left(\begin{array}{c}
D_{0 \tilde{1} \tilde{2} \tilde{3} 4} \\
D_{0 \tilde{2} \tilde{1} \tilde{3} 4} \\
D_{0 \tilde{2} \tilde{3} \tilde{1} 4} \\
D_{0 \tilde{1} \tilde{3} \tilde{2} 4} \\
D_{0 \tilde{3} \tilde{1} \tilde{2} 4} \\
D_{0 \tilde{3} \tilde{2} \tilde{1} 4}
\end{array}\right) \bullet\left(\check{D}_{0 \tilde{1} \tilde{2} \tilde{3} 4}, \check{D}_{0 \tilde{2} \tilde{1} \tilde{3} 4}, \check{D}_{0 \tilde{2} \tilde{z} \tilde{1} 4}, \check{D}_{0 \tilde{1} \tilde{3} \tilde{2} 4}, \check{D}_{0 \tilde{3} \tilde{1} \tilde{2} 4}, \check{D}_{0 \tilde{3} \tilde{2} \tilde{1} 4}\right) \\
& =\mathrm{F}(034) I_{034}^{12} .
\end{aligned}
$$


All data needed are evaluated already; we repeat them as follows. The action of the half-turn $H(03): D_{034}^{12} \rightarrow D_{304}^{12}$ is given by

$$
\begin{aligned}
& D_{0 \tilde{1} \tilde{2} 34} \rightarrow r_{0123} D_{3 \tilde{2} \tilde{1} 04}, \\
& D_{0 \tilde{2} \tilde{1} 34} \rightarrow r_{0213} D_{3 \tilde{1} \tilde{2} 04}, \\
& D_{0 \tilde{2} 3 \tilde{1} 4} \rightarrow-r_{023}\left(D_{3 \tilde{2} 0 \tilde{1} 4}+r_{01} D_{3 \tilde{2} \tilde{1} 04}+r_{01} r_{21} D_{3 \tilde{1} \tilde{2} 04}\right), \\
& D_{0 \tilde{1} 3 \tilde{2} 4} \rightarrow-r_{013}\left(D_{3 \tilde{1} 0 \tilde{2} 4}+r_{02} D_{3 \tilde{1} \tilde{2} 04}+r_{02} r_{12} D_{3 \tilde{2} \tilde{1} 04}\right), \\
& D_{03 \tilde{1} \tilde{2} 4} \rightarrow r_{03}\left(D_{30 \tilde{1} \tilde{2} 4}+r_{01} D_{3 \tilde{1} 0 \tilde{2} 4}+r_{01} r_{02} D_{3 \tilde{1} \tilde{2} 04}\right), \\
& D_{03 \tilde{2} \tilde{1} 4} \rightarrow r_{03}\left(D_{30 \tilde{2} \tilde{1} 4}+r_{02} D_{3 \tilde{2} 0 \tilde{1} 4}+r_{02} r_{01} D_{3 \tilde{2} \tilde{3} 04}\right),
\end{aligned}
$$

the full-turn $F(03): D_{034}^{12} \rightarrow D_{034}^{12}$ by the matrix $\mathrm{F}(03)=\mathrm{H}(03) \mathrm{H}(30)$ :

$$
\left(\begin{array}{cccccc}
c_{0123} & 0 & 0 & 0 & 0 & 0 \\
0 & c_{0213} & 0 & 0 & 0 & 0 \\
-c_{023} r_{31} r_{21} d_{01} & -c_{023} r_{31} d_{01 \cdot 21} & c_{023} & 0 & 0 & 0 \\
-c_{013} r_{32} d_{02 \cdot 12} & -c_{013} r_{32} r_{12} d_{02} & 0 & c_{013} & 0 & 0 \\
-c_{03} r_{31} r_{32} d_{01} & c_{03} c_{01} r_{123} d_{02} & 0 & -c_{03} r_{31} d_{01} & c_{03} & 0 \\
c_{03} c_{02} r_{213} d_{01} & -c_{03} r_{32} r_{31} d_{02} & -c_{03} r_{32} d_{02} & 0 & 0 & c_{03}
\end{array}\right) .
$$

The full-turn matrix $F(34)$ can be expressed in a similar way:

$$
\left(\begin{array}{cccccc}
c_{34} & 0 & 0 & -c_{34} r_{23} d_{24} & -c_{34} r_{23} r_{13} d_{24} & c_{34} c_{24} r_{123} d_{14} \\
0 & c_{34} & -c_{34} r_{13} d_{14} & 0 & c_{34} c_{14} r_{123} d_{24} & -c_{34} r_{13} r_{23} d_{14} \\
0 & 0 & c_{314} & 0 & -c_{314} r_{23} r_{21} d_{24} & -c_{314} r_{23} d_{24 \cdot 21} \\
0 & 0 & 0 & c_{324} & -c_{324} r_{13} d_{14 \cdot 12} & -c_{324} r_{13} r_{12} d_{14} \\
0 & 0 & 0 & 0 & c_{3124} & 0 \\
0 & 0 & 0 & 0 & 0 & c_{3214}
\end{array}\right) .
$$

Since they are triangular matrices,

$$
\mathrm{F}(034)=-(\mathrm{F}(03)-\mathrm{id})^{-1}-\mathrm{id}-(\mathrm{F}(34)-\mathrm{id})^{-1}
$$


can be computed without much difficulty as

$$
\begin{array}{ccc}
\frac{d_{0123 \cdot 34}}{d_{0123} d_{34}} & 0 & 0 \\
0 & \frac{d_{0123 \cdot 34}}{d_{0123} d_{34}} & \frac{c_{34} r_{13} d_{14}}{d_{34} d_{134}} \\
\frac{c_{023} r_{13} r_{12} d_{01}}{d_{023} d_{0123}} & \frac{c_{023} r_{13} d_{01 \cdot 12}}{d_{023} d_{0123}} & \frac{d_{023 \cdot 314}}{d_{023} d_{314}} \\
\frac{c_{013} r_{23} d_{02 \cdot 12}}{d_{013} d_{0123}} & \frac{c_{013} r_{23} r_{12} d_{02}}{d_{013} d_{0123}} & 0 \\
\frac{d_{01} d_{013 \cdot 02 \cdot 12} c_{03} r_{13} r_{23}}{d_{03} d_{013} d_{0123}} & -\frac{r_{123} d_{02} d_{03 \cdot 13} c_{01} c_{03}}{d_{03} d_{013} d_{0123}} & 0 \\
-\frac{r_{123} d_{01} d_{23 \cdot 03} c_{02} c_{03}}{d_{03} d_{023} d_{0123}} & \frac{d_{02} d_{023 \cdot 01 \cdot 12} c_{03} r_{13} r_{23}}{d_{03} d_{023} d_{0123}} & \frac{c_{03} r_{23} d_{02}}{d_{03} d_{023}}
\end{array}
$$

$\left.\begin{array}{ccc}\frac{c_{34} r_{23} d_{24}}{d_{34} d_{234}} & \frac{d_{24} d_{234 \cdot 12 \cdot 14} c_{34} r_{23} r_{13}}{d_{34} d_{234} d_{1234}} & -\frac{r_{123} d_{14} d_{23 \cdot 34} c_{24} c_{34}}{d_{34} d_{234} d_{1234}} \\ 0 & -\frac{r_{123} d_{24} d_{13 \cdot 34} c_{34} c_{14}}{d_{34} d_{134} d_{1234}} & \frac{d_{14} d_{134 \cdot 12 \cdot 24} c_{34} r_{23} r_{13}}{d_{34} d_{134} d_{1234}} \\ 0 & \frac{c_{134} r_{23} r_{12} d_{24}}{d_{134} d_{1234}} & \frac{c_{134} r_{23} d_{24 \cdot 12}}{d_{134} d_{1234}} \\ \frac{d_{013 \cdot 324}}{d_{013} d_{324}} & \frac{c_{234} r_{13} d_{14 \cdot 12}}{d_{234} d_{1234}} & \frac{c_{234} r_{13} r_{12} d_{14}}{d_{234} d_{1234}} \\ \frac{c_{03} r_{13} d_{01}}{d_{03} d_{013}} & \frac{d_{03 \cdot 3124}}{d_{03} d_{3124}} & 0 \\ 0 & 0 & \frac{d_{03 \cdot 3214}}{d_{03} d_{3214}}\end{array}\right)$.

The intersection matrix $I_{034}^{12}$ can be computed by

$$
I_{034}^{12}=\left(\begin{array}{cc}
\mathrm{F}(023) & \mathrm{G}(23) \\
\mathrm{G}(32) & \mathrm{F}(324)
\end{array}\right)\left(\begin{array}{cc}
I_{0234}^{1} & 0 \\
0 & I_{0324}^{1}
\end{array}\right)
$$


where

$$
I_{0234}^{1}=\left(\begin{array}{ccc}
-\frac{d_{01 \cdot 12}}{d_{01} d_{12}} & \frac{r_{12}}{d_{12}} & 0 \\
\frac{r_{21}}{d_{21}} & -\frac{d_{21 \cdot 13}}{d_{21} d_{13}} & \frac{r_{13}}{d_{13}} \\
0 & \frac{r_{31}}{d_{31}} & -\frac{d_{31 \cdot 14}}{d_{31} d_{14}}
\end{array}\right), \quad I_{0324}^{1}: \text { exchange } 2 \text { and } 3,
$$

and the matrix-representation of the operators

$$
F(023): D_{0234}^{1} \rightarrow D_{0234}^{1}, \quad G(23)=(F(23)-\mathrm{id})^{-1} H(23): D_{0234}^{1} \rightarrow D_{0324}^{1}
$$

are given as follows.

$$
\begin{aligned}
& H(02) \quad H(20) \\
& D_{0 \tilde{1} 234} \rightarrow-r_{012} D_{2 \tilde{1} 034} \rightarrow c_{012} D_{0 \tilde{1} 234}, \\
& D_{02 \tilde{1} 34} \rightarrow r_{02}\left(D_{20134}+r_{01} D_{0 \tilde{1} 234}\right) \rightarrow c_{02} D_{02 \tilde{1} 34}-c_{02} r_{21} d_{01} D_{0 \tilde{1} 234}, \\
& D_{023 \tilde{1} 4} \rightarrow r_{02} D_{203 \tilde{1} 4} \rightarrow c_{02} D_{023 \tilde{1} 4}, \\
& H(23) \quad H(32) \\
& D_{0 \tilde{1} 234} \rightarrow r_{23}\left(D_{0 \tilde{1} 324}+r_{13} D_{03 \tilde{1} 24}\right) \rightarrow c_{23} D_{0 \tilde{1} 234}-c_{23} r_{12} d_{13} D_{02 \tilde{1} 34}, \\
& D_{02 \tilde{1} 34} \rightarrow-r_{213} D_{03 \tilde{1} 24} \rightarrow c_{213} D_{02 \tilde{1} 34}, \\
& D_{023 \tilde{1} 4} \rightarrow r_{23}\left(D_{032 \tilde{1} 4}+r_{12} D_{03 \tilde{1} 24}\right) \rightarrow c_{23} D_{023 \tilde{1} 4}-c_{23} r_{31} d_{12} D_{02 \tilde{1} 34}, \\
& \mathrm{~F}(023)=\left(\begin{array}{ccc}
d_{012} & 0 & 0 \\
-c_{02} r_{12} d_{01} & d_{02} & 0 \\
0 & 0 & d_{02}
\end{array}\right)^{-1}+\mathrm{id}+\left(\begin{array}{ccc}
d_{23} & -c_{23} r_{12} d_{13} & 0 \\
0 & d_{123} & 0 \\
0 & -c_{23} r_{31} d_{12} & d_{23}
\end{array}\right)^{-1} \\
& =\left(\begin{array}{ccc}
\frac{d_{012 \cdot 23}}{d_{012} d_{23}} & \frac{c_{23} r_{12} d_{13}}{d_{23} d_{123}} & 0 \\
\frac{c_{02} r_{21} d_{01}}{d_{012} d_{02}} & \frac{d_{02 \cdot 123}}{d_{02} d_{123}} & 0 \\
0 & \frac{c_{23} r_{31} d_{12}}{d_{123} d_{23}} & \frac{d_{02 \cdot 23}}{d_{02} d_{23}}
\end{array}\right),
\end{aligned}
$$




$$
\begin{aligned}
-G(23) & =(\mathrm{H}(23) \mathrm{H}(32)-\mathrm{id})^{-1} \mathrm{H}(23)=\left\{\mathrm{H}(32)-\mathrm{H}(23)^{-1}\right\}^{-1} \\
& =\left(\begin{array}{ccc}
r_{32}-\frac{1}{r_{23}} & r_{32} r_{12}-\frac{1}{r_{23} r_{21}} & 0 \\
0 & -r_{32} r_{31} r_{12}+\frac{1}{r_{23} r_{21} r_{13}} & 0 \\
0 & r_{32} r_{13}-\frac{1}{r_{23} r_{13}} & r_{32}-\frac{1}{r_{23}}
\end{array}\right)^{-1} \\
& =\left(\begin{array}{ccc}
\frac{r_{23}}{d_{23}} & \frac{d_{23 \cdot 21} r_{123}}{r_{12} d_{23} d_{123}} & 0 \\
0 & -\frac{r_{123}}{d_{123}} & 0 \\
0 & \frac{d_{23 \cdot 13} r_{123}}{r_{13} d_{23} d_{123}} & \frac{r_{23}}{d_{23}}
\end{array}\right) .
\end{aligned}
$$

The matrix-representation of the operators

$$
F(324): D_{0324}^{1} \rightarrow D_{0324}^{1}, \quad G(32)=(F(32)-\mathrm{id})^{-1} H(32): D_{0324}^{1} \rightarrow D_{0234}^{1}
$$

are given as follows.

$$
\begin{aligned}
& H(32) \\
& H(23) \\
& D_{0 \tilde{1} 324} \rightarrow r_{32}\left(D_{0 \tilde{1} 234}+r_{12} D_{02 \tilde{1} 34}\right) \rightarrow c_{32} D_{0 \tilde{1} 324}-c_{32} r_{13} d_{12} D_{03 \tilde{1} 24}, \\
& D_{03 \tilde{1} 24} \rightarrow-r_{312} D_{02 \tilde{1} 34} \rightarrow c_{312} D_{03 \tilde{1} 24}, \\
& D_{032 \tilde{1} 4} \rightarrow r_{32}\left(D_{023 \tilde{1} 4}+r_{13} D_{02 \tilde{1} 34}\right) \rightarrow c_{32} D_{032 \tilde{1} 4}-c_{32} r_{21} d_{31} D_{03 \tilde{1} 24}, \\
& H(24) \\
& D_{01324} \rightarrow r_{24} D_{01 \tilde{3} 42} \rightarrow c_{24} D_{01 \tilde{3} 24}, \\
& D_{03 \tilde{1} 24} \rightarrow r_{24}\left(D_{03 \tilde{1} 42}+r_{14} D_{034 \tilde{1} 2}\right) \quad \rightarrow \quad c_{24} D_{03 \tilde{1} 24}-c_{24} r_{12} d_{14} D_{032 \tilde{1} 4}, \\
& D_{032 \tilde{1} 4} \rightarrow-r_{214} D_{034 \tilde{1} 2} \rightarrow c_{214} D_{032 \tilde{1} 4}, \\
& \mathrm{~F}(324)=\left(\begin{array}{ccc}
d_{23} & -c_{23} r_{13} d_{12} & 0 \\
0 & d_{312} & 0 \\
0 & -c_{32} r_{21} d_{31} & d_{23}
\end{array}\right)^{-1}+\mathrm{id}+\left(\begin{array}{ccc}
d_{24} & 0 & 0 \\
0 & d_{24} & -c_{24} r_{12} d_{14} \\
0 & 0 & d_{214}
\end{array}\right)^{-1} \\
& =\left(\begin{array}{ccc}
\frac{d_{23 \cdot 24}}{d_{23} d_{24}} & \frac{c_{23} r_{13} d_{12}}{d_{23} d_{312}} & 0 \\
0 & \frac{d_{312 \cdot 24}}{d_{312} d_{24}} & \frac{c_{24} r_{12} d_{14}}{d_{24} d_{214}} \\
0 & \frac{c_{32} r_{21} d_{31}}{d_{312} d_{23}} & \frac{d_{23 \cdot 214}}{d_{23} d_{214}}
\end{array}\right),
\end{aligned}
$$

G(32) being obtained from $\mathrm{G}(23)$ by exchanging 2 and 3 .

Now we put

$$
c_{01}=c_{02}=c_{03}=a, \quad c_{14}=c_{24}=c_{34}=c, \quad r_{12}=r_{23}=r_{31}=g .
$$


The vertical sums of the entries of $\mathrm{F}(034)$ are $\left(f_{1}, f_{1}, f_{2}, f_{2}, f_{3}, f_{3}\right)$, where

$$
\left(f_{1}, f_{2}, f_{3}\right):=\left(1-a g^{2} b\right)\left(\frac{1}{\left(1-a g^{2}\right)(1-b)}, \frac{1}{(1-a g)(1-g b)}, \frac{1}{(1-a)\left(1-g^{2} b\right)}\right) .
$$

Let us honestly compute

$$
\left(h_{1}, \ldots, h_{6}\right):=\left(f_{1}, f_{1}, \ldots, f_{3}\right)\left(\begin{array}{cc}
\mathrm{F}(023) & \mathrm{G}(23) \\
\mathrm{G}(32) & \mathrm{F}(324)
\end{array}\right) .
$$

Then we have

$$
h_{1}=-\frac{\left(1-a g^{2} b\right)\left(1-a g^{3} b\right)}{(1-a g)\left(1-a g^{2}\right)(1-b)(1-g b)(g+1)},
$$

$$
\begin{gathered}
h_{2}= \\
-\frac{\left(1-a g^{2} b\right)\left(1-a g^{3} b\right)\left(a b g^{4}-(a+b) g^{3}+2 a b g^{3}-(a+b) g^{2}-(a+b) g+2 g+1\right)}{(1-a)(1-a g)\left(1-a g^{2}\right)(1-b)(1-g b)\left(1-g^{2} b\right)(g+1)\left(g^{2}+g+1\right)}, \\
h_{3}=-\frac{\left(1-a g^{2} b\right)\left(1-a g^{3} b\right)}{(1-a)(1-a g)(1-g b)\left(1-g^{2} b\right)(g+1)}, \\
h_{4}=h_{1}, \quad h_{5}=h_{2}, \quad h_{6}=h_{3} .
\end{gathered}
$$

On the other hand, the horizontal sums of the entries of $I_{0234}^{1}$ are

$$
\left(I_{1}, I_{2}, I_{3}\right)=\frac{1}{g+1}\left(\frac{a g+1}{1-a}, 1-g, \frac{g b+1}{1-b}\right) .
$$

We have

$$
\begin{aligned}
& \left(1-g^{2} b\right)\left(g^{2}+g+1\right)(a g+1) \\
& \quad+\left(a c g^{4}-(a+b) g^{3}+2 a b g^{3}-(a+b) g^{2}-(a+b) g+2 g+1\right)(1-g) \\
& \quad+\left(1-a g^{2}\right)\left(g^{2}+g+1\right)(g b+1)=3\left(1-a g^{4} b\right)(g+1),
\end{aligned}
$$

and so the sum $J_{3}$ can be computed, and factors as

$$
J_{3}=-\frac{3 !\left(1-g^{4} a b\right)\left(1-g^{3} a b\right)\left(1-g^{2} a b\right)}{(1-a)(1-a g)\left(1-a g^{2}\right)(1-b)(1-g b)\left(1-g^{2} b\right)(g+1)\left(g^{2}+g+1\right)},
$$

which agrees with the result obtained geometrically in [MiY2].

5. General theory on the twisted (co)homology groups on fibred spaces ([OST)]. Let $\pi: X \rightarrow B$ be a fibre bundle, and $\mathcal{L}$ a local system on $X$. Assume pure (co)dimensionality of the total space and the fibres:

$$
\begin{array}{ccc}
H_{i}(X, \mathcal{L})=0, & H^{i}(X, \mathcal{L})=0, & \text { if } \quad i \neq n:=\operatorname{dim}_{C} X, \\
H_{i}\left(\pi^{-1}(b), \iota_{b}^{*} \mathcal{L}\right)=0, & H^{i}\left(\pi^{-1}(b), \iota_{b}^{*} \mathcal{L}\right)=0, & \text { if } \quad i \neq f:=\operatorname{dim}_{C} \pi^{-1}(b),
\end{array}
$$


where $\iota_{b}: \pi^{-1}(b) \rightarrow X$ is the inclusion map. Then we have the natural isomorphisms

$$
H_{n}(X, \mathcal{L}) \cong H_{n-f}\left(B, \mathcal{H}_{f}\right), \quad H^{n}(X, \mathcal{L}) \cong H^{n-f}\left(B, \mathcal{H}^{f}\right),
$$

where $\mathcal{H}_{f}$ and $\mathcal{H}^{f}$ are local systems on $B$ defined as the locally constant sheaves of germs of locally flat sections of the bundles

$$
\bigcup_{b \in B} H_{f}\left(\pi^{-1}(b), \iota_{b}^{*} \mathcal{L}\right) \text { and } \bigcup_{b \in B} H^{f}\left(\pi^{-1}(b), \iota_{b}^{*} \mathcal{L}\right),
$$

respectively.

Let $\gamma \in H_{n}(X, \mathcal{L})$ be represented by the finite sum

$$
\sum a_{i} \delta_{i} \otimes u_{i}, \quad a_{i} \in \boldsymbol{C},
$$

where $\delta_{i} \in H_{n-f}(B, \boldsymbol{Z})$, and $u_{i}$ is a section of $\mathcal{H}_{f}$ along the support $\left|\delta_{i}\right|$ of $\delta_{i}$. Let $\gamma^{\prime} \in$ $H_{n}^{\text {lf }}(X, \check{\mathcal{L}})$ be represented by the locally finite $\operatorname{sum} \sum a_{i}^{\prime} \delta_{i}^{\prime} \otimes u_{i}^{\prime}$, where $\delta_{i}^{\prime} \in H_{n-f}^{\text {lf }}(B, \boldsymbol{Z})$, and $u_{i}^{\prime}$ is a section of $\check{\mathcal{H}}_{f}^{\text {lf }}$, which is defined as the locally constant sheaf of germs of horizonatal sections of the bundle

$$
\bigcup_{b \in B} H_{f}^{\mathrm{lf}}\left(\pi^{-1}(b), \iota_{b}^{*} \check{\mathcal{L}}\right)
$$

along the support $\left|\delta_{i}^{\prime}\right|$ of $\delta_{i}^{\prime}$. Then the intersection number $\gamma \cdot \gamma^{\prime}$ is equal to

$$
\sum_{\{b\}=\left|\delta_{i}\right| \cap\left|\delta_{j}\right|} a_{i} a_{j}^{\prime}\left(\delta_{i} \cdot \delta^{\prime}\right)(b)\left(u_{i} \cdot u_{j}^{\prime}\right)(b),
$$

where $\left(\delta_{i} \cdot \delta_{j}^{\prime}\right)(b)$ is the topological intersection number at $p$, and $\left(u_{i} \cdot u_{j}^{\prime}\right)(b)$ is defined by the intersection pairing between $H_{f}\left(\pi^{-1}(b), \iota_{b}^{*} \mathcal{L}\right)$ and $H_{f}^{\mathrm{lf}}\left(\pi^{-1}(b), \iota_{b}^{*} \breve{\mathcal{L}}\right)$.

Let $f \in H_{c}^{n}(X, \mathcal{L})$ be represented by the finite sum

$$
\sum a_{i} g_{i} \otimes v_{i}, \quad a_{i} \in \boldsymbol{C},
$$

where $g_{i}$ is a compactly supported $(n-f)$-form on $B$ and $v_{i}$ is a section of $\mathcal{H}_{c}^{f}$, that is, $v_{i}$ is a compactly supported $f$-form with values in $\mathcal{L}$ and with parameter $b$ on the generic fibre. Let $f^{\prime} \in H^{n}(X, \check{\mathcal{L}})$ be represented by the finite sum $\sum a_{i}^{\prime} g_{i}^{\prime} \otimes v_{i}^{\prime}$, where $g_{i}^{\prime}$ is an $(n-f)$-form on $B$ and $v_{i}$ is a section of $\check{\mathcal{H}}^{f}$, that is, $v_{i}$ is an $f$-form with values in $\check{\mathcal{L}}$ and with parameter $b$ on the generic fibre. The intersection number $f \cdot f^{\prime}$ is equal to

$$
\sum a_{i} a_{j}^{\prime} \int\left(v_{i} \cdot v_{j}^{\prime}\right)(b) g_{i} \wedge g_{j}^{\prime},
$$

where $\left(v_{i} \cdot v_{j}\right)(b)$ is defined by the intersection pairing between $H^{f}\left(\pi^{-1}(b), \iota_{b}^{*} \mathcal{L}\right)$ and $H^{f}\left(\pi^{-1}(b), \iota_{b}^{*} \check{\mathcal{L}}\right)$.

The de Rham theorem and the Fubini theorem imply the assersion for cohomology groups. The intersection form for homology groups is defined ([KY]) through that of cohomology groups (this is the compatibility of the two intersection theories). So the assersion for the cohomology groups leads to that for the homology groups. 


\section{REFERENCES}

[KY] M. KITA AND M. YoshidA, Intersection theory for twisted cycles, Math. Nach. 166 (1994), $287-304$.

[MiY1] K. Mimachi And M. YoshidA, Intersection numbers of twisted cycles and the correlation functions of the conformal field theory, Comm. Math. Phys. 234 (2003), 339-358.

[MiY2] K. Mimachi AND M. YoshidA, Intersection numbers of twisted cycles with the Selberg integral and an application to the conformal field theory, Comm. Math. Phys. 250 (2004), 23-25.

[Oh] K. OHARA, Intersection forms on twisted cohomology groups associated with Selberg-type integrals, preprint 2002.

[OST] K. Ohara, Y. Sugiki And N. TAKAYAma, Quadratic relations for generalized hypergeometric functions ${ }_{p} F_{p-1}$, Funk. Ekvac. 46 (2003), 213-252.

[Yo] M. Yoshida, Hypergeometric Functions, My Love, Vieweg Verlag, Wiesbaden, 1997.

DEPARTMENT OF MATHEMATICS

TOKYO Institute OF TECHNOLOGY

TOKYO 152-8551

JAPAN

E-mail address: mimachi@math.titech.ac.jp

DEPARTMENT OF MATHEMATICS

KYUSHU UNIVERSITY

FUKUOKA 810-8560

JAPAN

E-mail address: myoshida@math.kyushu-u.ac.jp
DEPARTMENT OF COMPUTATIONAL SCIENCE

KANAZAWA UNIVERSITY

KANAZAWA 920-1192

JAPAN

E-mail address: ohara@air.s.kanazawa-u.ac.jp 